Новая классификация холестеатомы EAONO/JOS

и хирургических вмешательств на среднем ухе SAMEO-ATO: актуальность и клиническая значимость

\author{
Е.В. Пчеленок ${ }^{\bowtie}$ О.Ю. Тарасова, С.Я. Косяков
}

ФГБОУ ДПО «Российская медицинская академия непрерывного профессионального образования» Минздрава России, Москва, Россия

\begin{abstract}
Аннотация
Холестеатома среднего уха (ХСУ) является распространенной проблемой современной отологии. Европейская академия отологии и нейроотологии совместно с Японским отологическим обществом (EAONO/JOS) в 2017 г. опубликовали консенсусные рекомендации по определению, классификации и стадиям ХСУ. В 2018 г. под эгидой Международного общества по оценке результатов в отологии (The International Otology Outcome Group) представлена классификация хирургических вмешательств на среднем ухе. Ведущей проблемой хирургии ХСУ остается высокая частота рецидивирования, которая составляет от 5 до $32 \%$ в зависимости от типа хирургического вмешательства. Цель статьи - представить описание новой классификации XCУ EAONO/JOS и хирургических вмешательств на среднем ухе SAMEO-ATO с анализом их возможной клинической значимости для определения риска рецидивирования холестеатомы, а также прогноза в отношении восстановления слуха после операции.
\end{abstract}

Ключевые слова: холестеатома среднего уха, классификация холестеатомы, стадии холестеатомы, хирургия среднего уха, клиническая значимость

Для цитирования: Пчеленок Е.В., Тарасова О.Ю., Косяков С.Я. Новая классификация холестеатомы EAONO/JOS и хирургических вмешательств на среднем ухе SAMEO-ATO: актуальность и клиническая значимость. Consilium Medicum. 2021;23(12):956-960. DOI: 10.26442/20751753.2021.12.201236

REVIEW

\title{
New classification of EAONO/JOS cholesteatoma and SAMEO-ATO middle ear surgery: relevance and clinical significance
}

\author{
Ekaterina V. Pchelenok ${ }^{\bowtie}$, Olga Yu. Tarasova, Sergey Ya. Kosyakov \\ Russian Medical Academy of Continuous Professional Education, Moscow, Russia
}

Abstract

Middle ear cholesteatoma (MEC) is a common problem of modern otology. The European Academy of Otology and Neurootology in cooperation with the Japanese Otological Society (EAONO/JOS) published consensus recommendations on the definition, classification and stages of MEC in 2017. In 2018, the classification of middle ear surgery was presented under the auspices of the International Otology Outcome Group. The leading problem of MEC surgery remains the high recurrence rate, which ranges from 5 to $32 \%$ depending on the type of surgical intervention. The main purpose of this article is to describe the new EAONO/JOS classification of MEC and SAMEO-ATO middle ear surgical interventions with an analysis of their possible clinical significance in determining the risk of cholesteatoma recurrence as well as the prognosis for hearing recovery after surgery.

Keywords: middle ear cholesteatoma, cholesteatoma classification, stages of cholesteatoma, middle ear surgery, clinical significance For citation: Pchelenok EV, Tarasova OYu, Kosyakov SYa. New classification of EAONO/JOS cholesteatoma and SAMEO-ATO middle ear surgery: relevance and clinical significance. Consilium Medicum. 2021;23(12):956-960. DOI: 10.26442/20751753.2021.12.201236

\section{Введение}

Холестеатома среднего уха (ХСУ) является одной из наиболее актуальных проблем современной отологии, что связано с высокой распространенностью, значительной частотой развития экстра- и интракраниальных осложнений, трудностями диагностики и лечения $[1,2]$.Ежегодная заболеваемость холестеатомой оценивается в 9,2 на 100 тыс. человек у взрослых и 3 на 100 тыс. у детей. По данным другого исследования, заболеваемость составляет около 6 случаев на 100 тыс. населения [3].

Согласно современным представлениям в большинстве случаев холестеатома является осложнением хронического гнойного среднего отита [4]. С гистологической точки зрения холестеатома состоит из 3 слоев:

1) собственно холестеатомные массы (детрит);

2) матрикс (пролиферирующие эпидермальные кератиноциты);

3) периматрикс (соединительнотканный слой, содержащий кровеносные сосуды, фибробласты, лимфоциты, плазматические клетки и др.) $[2,5,6]$.

Несмотря на длительную историю изучения и многочисленные теории патогенеза (теория инвагинации, метаплазии, миграционная теория, теория ретракции и пролиферации и др.), продолжаются исследования по

\section{Информация об авторах / Information about the authors}

Пчеленок Екатерина Витальевна - канд. мед. наук, доц. каф. оториноларингологии ФГБОУ ДПО РМАНПО.

E-mail: epchelenok@yandex.ru; ORCID: 0000-0003-1021-5403

Тарасова Ольга Юрьевна - аспирант каф. оториноларингологии ФГБОУ ДПО РМАНПО. E-mail: olya.tar@inbox.ru; ORCID: 0000-0002-6986-9260

Косяков Сергей Яковлевич - д-р мед. наук, проф., зав. каф. оториноларингологии ФГБОУ ДПО РМАНПО.

E-mail: Serkosykov@yandex.ru; ORCID: 0000-0001-7242-2593
Ekaterina V. Pchelenok - Cand. Sci. (Med.), Russian Medical Academy of Continuous Professional Education. E-mail: epchelenok@yandex.ru; ORCID: 0000-0003-1021-5403

Olga Yu. Tarasova - Graduate Student, Russian Medical Academy of Continuous Professional Education. E-mail: olya.tar@inbox.ru; ORCID: 0000-0002-6986-9260

Sergey Ya. Kosyakov - D. Sci. (Med.), Prof., Russian Medical Academy of Continuous Professional Education. E-mail: Serkosykov@yandex.ru; ORCID: 0000-0001-7242-2593 
выявлению точных причин и механизмов развития холестеатомы [6].

Основными клиническими проявлениями холестеатомы являются оторея и снижение слуха. Важно отметить, что в большинстве случаев наблюдается поздняя диагностика холестеатомы [3]. Так, примерно в $1 / 3$ случаев диагноз ставится спустя 6 лет и более после появления первых симптомов заболевания, что определяет достаточно высокую частоту развития осложнений. Примерно в $80 \%$ случаев при холестеатоме наблюдается деструкция слуховых косточек [3]. Также при холестеатоме могут развиваться головокружение, парез лицевого нерва, различные внутричерепные осложнения, включая эпи- и субдуральный абсцесс, менингит и др. Все это определяет важность своевременной диагностики и лечения холестеатомы.

В настоящее время хирургическое лечение остается единственной доступной опцией при лечении пациентов с ХСУ. Хирургическое лечение направлено на полное удаление патологического процесса с целью предотвращения развития осложнений и улучшения слуха. Ведущей проблемой хирургического лечения ХСУ является высокая частота рецидивирования, составляющая от 5 до $32 \%$ в зависимости от различных типов хирургического лечения $[7,8]$.

В последние годы активно разрабатываются различные системы классификации и стадирования холестеатомы [8]. Авторы пришли к выводу, что данные системы могут быть использованы для определения риска рецидивирования и прогноза в отношении восстановления слуха после операций, а также для корректного сравнения результатов клинических исследований с применением различных типов хирургических вмешательств. К настоящему времени предложено достаточно большое количество классификаций и систем стадирования холестеатомы различной сложности, которые позволяют учитывать различные факторы, однако ни одна из них полностью не отвечает практическим и научным целям [9-16]. При описании холестеатомы авторы могут использовать различные термины, которые не всегда имеют однозначную трактовку. Эта же проблема касается и технических аспектов проведения оперативных вмешательств [17]. Кроме того, необходимо отметить, что до настоящего времени, несмотря на неоспоримость эффективности хирургического лечения холестеатомы, крайне мало данных о сравнительной эффективности различных методов, которые были бы получены в качественных рандомизированных клинических исследованиях. Выбор метода оперативного лечения холестеатомы основывается преимущественно на индивидуальных особенностях пациента и опыте конкретного хирурга, при этом сравнение результатов оперативного лечения, проведенного в разных центрах, затруднено отсутствием общепринятой терминологии для описания подобного рода вмешательств [6]. Все это определяет большую актуальность разработки новых классификаций холестеатомы и хирургических вмешательств на среднем ухе.

\section{Новая классификация холестеатомы EAONO/JOS}

В 2017 г. опубликованы совместные консенсусные рекомендации EAONO/JOS (Европейская академия отологии и нейроотологии/Японское отологическое общество) по определению, классификации и стадиям ХСУ [6]. Разработке этих рекомендаций предшествовала публикация рекомендаций по холестеатоме, изданных EAONO в 2015 г., и рекомендаций JOS, опубликованных в 2017 г. [14, 15]. При разработке консенсусных рекомендаций EAONO/JOS преследовали следующие цели:

1) сформулировать определение холестеатомы для уточнения терминологии, используемой при описании этого образования;

2) разработать классификацию ХСУ для более корректного сравнения результатов применения хирургических методов лечения;
3) разработать систему стадирования ХСУ, которая бы отражала тяжесть заболевания, сложность хирургического удаления холестеатомы и возможность последующего восстановления нормальной функции.

С методологической точки зрения разработка данных рекомендаций проходила в несколько этапов. Первоначально разрабатывался 1-й вариант документа при участии 6 европейских и 4 японских отологов. В последующем текст рекомендаций неоднократно обсуждался, в том числе в рамках 10-й Международной конференции по холестеатоме и хирургии уха в г. Эдинбурге (июнь 2016 г.), проходившей при участии более 1 тыс. делегатов из 54 стран, а также в рамках специально собранной консенсусной сессии. В дальнейшем 47 заинтересованных делегатов представили обратную связь по 3-й редакции документа; на этом этапе внесено еще несколько дополнительных незначительных изменений. В целом текст рекомендаций получил одобрение большинства специалистов, при этом предложенное определение холестеатомы получило одобрение в 98\% случаев, классификация - в 89\% случаев, предложенная система стадирования - в 75\% [6].

Согласно предложенному определению холестеатома представляет собой массу, состоящую из ороговевающего плоского эпителия, локализующуюся в барабанной и/или мастоидальной полости и субэпителиальной соединительной ткани, для которой характерно прогрессирующее накопление кератина с перифокальной воспалительной реакцией или без нее. Холестеатома состоит из остатков кератина, матрикса и периматрикса. В рекомендациях особенно подчеркивается, что патофизиологические механизмы развития холестеатомы остаются окончательно не изученными [6].

С точки зрения классификации консенсусные рекомендации сохранили подразделение холестеатомы на 2 основные категории: приобретенная и врожденная. Отдельно предложена категория «неклассифицируемая холестеатома».

Для приобретенной холестеатомы характерно развитие клинических проявлений, обусловленных ростом образования или сдавлением окружающих структур. Приобретенная холестеатома подразделяется на следующие основные варианты:

1. Холестеатома, развивающаяся из ретракционного кармана:

• ненатянутой части барабанной перепонки (БП) - pars flaccida;

- натянутой части БП - pars tensa;

- ненатянутой и натянутой частей БП.

2. Холестеатома, не связанная с формированием ретракционного кармана:

- вторичная в результате разрыва БП;

- возникающая после проведения отологических процедур (ятрогенная) или травмы.

При описании приобретенной холестеатомы необходимо учитывать наличие или отсутствие деструкции прилежащих структур, ретракции и/или перфорации БП, отореи, снижения слуха, а также результаты применения методов визуализации (компьютерной томографии/магнитно-резонансной томографии).

Врожденная холестеатома представляет собой эпидермоидную кисту, которая располагается медиальнее интактной БП. В большинстве случаев врожденная холестеатома располагается в передневерхнем квадранте среднего уха. Данная локализация врожденной холестеатомы является единственной из упомянутых в рекомендациях EAONO (2015 г.), однако в консенсусных рекомендациях EAONO/JOS также в качестве возможного варианта локализации врожденной холестеатомы отмечается задневерхний квадрант, а также допускается возможность и иной локализации. Предполагается, что врожденная холестеатома имеется уже при рождении, хотя и диагностируется, как правило, позже. Клинически врожденная холестеатома проявляется тугоухостью, в редких случаях - болевым синдромом. 
Рис. 1. Классификация ХCУ EAONO/JOS (адаптировано из [6])

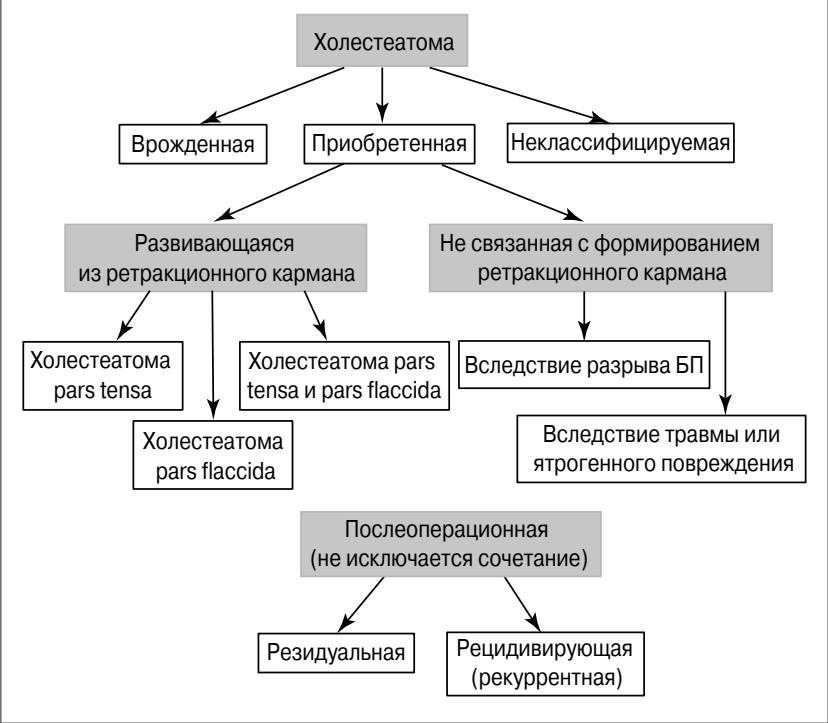

В качестве отдельной категории в классификации рассматривается рецидивирование холестеатомы после хирургических вмешательств, которое может быть представлено:

- резидуальной холестеатомой (возникает в результате неполного удаления матрикса);

- рецидивирующей (рекуррентной) холестеатомой (воз-

никает в результате реформирования ретракционного кармана после хирургического лечения).

Среди особенностей классификации EAONO/JOS необходимо отметить отказ от использования терминов «первично» и «вторично приобретенная холестеатома», поскольку они в большей степени имеют историческое значение. Также необходимо отметить внедрение термина «неклассифицируемая холестеатома» для тех случаев, когда невозможно точно определить врожденный или приобретенный характер образования. Данный термин, в частности, рекомендовано использовать вместо термина «подозрение на врожденную холестеатому».

Схематично предложенная классификация холестеатомы представлена на рис. 1.

Стадирование холестеатомы основано на анатомическом подразделении полости среднего уха на 4 части (рис. 2):

- труднодоступная область (S), которая в свою очередь делится на передний эпитимпанум, или протимпанум

(S1), и барабанный синус (S2);

- барабанная полость (T);

- аттик (А);

- сосцевидный отросток (M)

Стадирование XСУ EAONO/JOS применимо для 4 вариантов холестеатомы (холестеатома натянутой и ненатянутой частей БП, врожденная холестеатома и холестеатома вследствие перфорации БП) и подразумевает выделение 4 стадий:

Стадия I - холестеатома в месте возникновения (например, в области А для холестеатомы ненатянутой части БП или в области Т для холестеатомы натянутой части БП).

Стадия II - холестеатома с вовлечением 2 и более областей.

Стадия III - холестеатома с экстракраниальными осложнениями, включая парез лицевого нерва, лабиринтит, фистулу лабиринта, постаурикулярный абсцесс, абсцесс в области шеи и др.; на этой стадии также могут возникать деструкция стенки канала, крыши барабанной полости и адгезивный отит.

Стадия IV - холестеатома с интракраниальными осложнениями, к которым относятся гнойный менингит, эпидуральный и субдуральный абсцесс, абсцесс головного мозга, тромбоз венозных синусов и др.
Рис. 2. Разделение полости среднего уха по системе STAM (адаптировано из [6]).

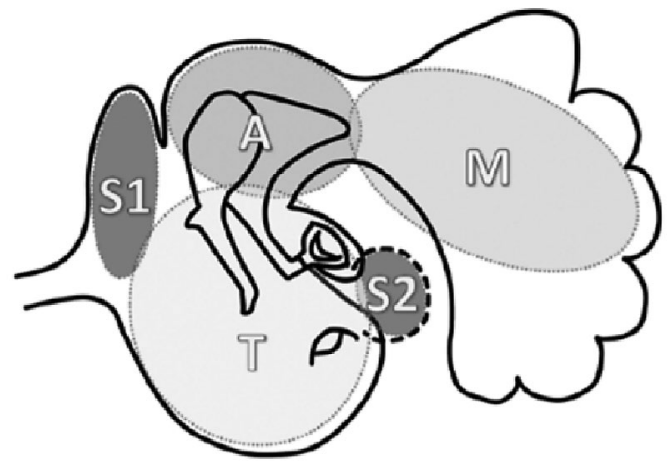

Следует отметить, что именно система стадирования ХСУ вызвала наиболее оживленные дебаты во время подготовки данных рекомендаций. В первую очередь речь идет о сложности системы стадирования. Относительно простые системы удобны для широкого практического применения. С другой стороны, более сложные и развернутые системы стадирования позволяют учесть больше потенциальных прогностических факторов. Предложенная классификация критиковалась за излишнюю простоту. На этапе обсуждения предложено несколько более сложных вариантов классификации, позволяющих, в частности, учесть деструкцию слуховых косточек, состояние слизистой оболочки и др. В проведенных к настоящему времени исследованиях показано, что многие факторы могут влиять на восстановление слуха после хирургического лечения холестеатомы, однако по этим дополнительным параметрам достичь консенсуса еще сложнее, так как для большинства факторов их прогностическое значение и влияние на исход оперативных вмешательств остаются недостаточно изученными [6].

Следует отметить, что к настоящему времени уже опубликован ряд исследований, в которых успешно применялась классификация EAONO/JOS (2017 г.). В первую очередь необходимо отметить результаты крупного исследования с включением более 1482 пациентов с ХСУ, в котором проводилась оценка валидности предложенной системы стадирования [18]. В данной работе на репрезентативной выборке пациентов показано, что большинство пациентов с холестеатомой имеют II (67\%) и I (22\%) стадии заболевания, значительно реже встречается III стадия (11\%) и крайне редко - IV (0,3\%). Авторы показали высокую внутри- и межэкспертную надежность определения стадии $(\kappa=0,8)$, хотя расхождения в определении стадии между различными экспертами встречались более чем в $10 \%$ случаев, что требует дополнительных исследований $[18,19]$.

В работе, проведенной М. Motegi и соавт. (2019 г.), подтверждено, что у большинства пациентов с ХСУ определяются II $(59,1 \%)$ и I $(31,8 \%)$ стадии, при этом при использовании на данной выборке пациентов предложенной ранее классификации JOS доля пациентов со II и I стадиями составила соответственно 50,0 и 40,9\% [20]. Основной причиной расхождения является выявленная у 9,1\% пациентов инвазия образования в барабанный синус, что позволило отнести эти случаи ко II стадии по EAONO/JOS. В исследовании, проведенном F. Ardic и соавт. (2020 г.), получены данные о большей частоте встречаемости пациентов с III стадией заболевания (31\%), при этом доля пациентов с I и II стадиями составила соответственно 39 и $28 \%$, с IV стадией - $2 \%$ [8].

M. Komori и соавт. (2018 г.) провели анализ практической ценности классификации и системы стадирования JOS холестеатомы pars flaccida, II стадию выявляли наиболее часто (71\% общего количества случаев), средний возраст пациентов составил 30-40 лет, при этом III стадия встречалась реже, возрастная группа - старше 40 лет [16]. Авторы 


\begin{tabular}{|c|c|}
\hline \multicolumn{2}{|r|}{ S-state of surgery (эman onepauuu) } \\
\hline S1 & Первичная операция \\
\hline$S 2 p$ & $\begin{array}{l}\text { Запланированное 2-е вмешательство } \\
\text { (или 2-й этап операции) }\end{array}$ \\
\hline $\mathrm{S} 2 \mathrm{r}$ & Ревизия (незапланированное 2-е вмешательство) \\
\hline \multicolumn{2}{|r|}{ 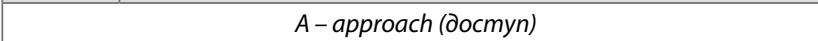 } \\
\hline A1 & Эндоскопический трансканальный \\
\hline $\mathrm{A} 2$ & Микроскопический трансканальный \\
\hline $\mathrm{A} 3$ & Эндауральный \\
\hline A4 & Ретроаурикулярный \\
\hline \multicolumn{2}{|r|}{ M-mastoidectomy (мастоидэктомия) } \\
\hline$M x$ & Мастоидэктомия не проводилась \\
\hline M1a & $\begin{array}{l}\text { Мастоидэктомия с сохранением стенки канала } \\
\text { (кортикальная мастоидэктомия) }\end{array}$ \\
\hline M1b & $\begin{array}{c}\text { Мастоидэктомия с сохранением стенки канала } \\
\text { (кортикальная мастоидэктомия) + задняя тимпанотомия }\end{array}$ \\
\hline $\mathrm{M} 2 \mathrm{a}$ & $\begin{array}{c}\text { Мастоидэктомия с удалением только верхнего щита } \\
\text { (scuttum) - аттикотомия }\end{array}$ \\
\hline $\mathrm{M} 2 \mathrm{~b}$ & $\begin{array}{c}\text { Мастоидэктомия с удалением верхнего щита } \\
\text { и задневерхней стенки канала (аттикоантротомия) }\end{array}$ \\
\hline $\mathrm{M} 2 \mathrm{c}$ & Мастоидэктомия с удалением всего канала \\
\hline $\mathrm{M} 1 \mathrm{a}+2 \mathrm{a}$ & $\begin{array}{l}\text { Мастоидэктомия с сохранением } \\
\text { стенки канала + аттикотомия }\end{array}$ \\
\hline$M 1 b+2 a$ & $\begin{array}{c}\text { Мастоидэктомия с сохранением } \\
\text { стенки канала + задняя тимпанотомия + аттикотомия }\end{array}$ \\
\hline M3a & $\begin{array}{c}\text { Субтотальная петрозэктомия с coxранением } \\
\text { слуховой капсулы (oticcapsula) }\end{array}$ \\
\hline M3b & $\begin{array}{c}\text { Субтотальная петрозэктомия с удалением слуховой капсулы } \\
\text { (oticcapsula) }\end{array}$ \\
\hline \multicolumn{2}{|r|}{$\begin{array}{c}\text { E- external ear canal reconstruction (реконструкция наружного } \\
\text { слухового прохода) }\end{array}$} \\
\hline Ex & Реконструкция не проводилась \\
\hline E1 & Реконструкция с применением мягких материалов \\
\hline E2 & Реконструкция с применением ригидных материалов \\
\hline \multicolumn{2}{|r|}{$\begin{array}{l}\text { O- obliteration of mastoid cavity (облитерачия ячеек } \\
\text { сосцевидного отростка) }\end{array}$} \\
\hline Ox & Облитерация не проводилась \\
\hline 01 & Частичная облитерация \\
\hline $\mathrm{O} 2$ & Полная облитерация \\
\hline \multicolumn{2}{|r|}{ A-access to middle ear (доступ к среднему уху) } \\
\hline$A x$ & Без удаления кости \\
\hline A1 & Расширение задней порции барабанной борозды \\
\hline A2 & $\begin{array}{l}\text { Частичное или круговое расширение костного канала } \\
\text { (каналопластика) }\end{array}$ \\
\hline A3 & Полная каналопластика с пластикой мягких тканей \\
\hline \multicolumn{2}{|r|}{$T$-tympanic membrane (БП) } \\
\hline Tx & Восстановление (пластика) БП не проводилось \\
\hline Tn & БП сохранена \\
\hline T1 & Укрепление интактной БП (reinforcement) \\
\hline T2 & Частичная пластика БП \\
\hline T3 & Полная пластика БП \\
\hline \multicolumn{2}{|r|}{ O-ossicular chain (цепь слуховых косточек) } \\
\hline Ox & Реконструкция не проводилась \\
\hline On & Цепь слуховых косточек сохранена \\
\hline Osi & Реконструкция между наковальней и головкой стремени \\
\hline Osm & Реконструкция между молоточком и головкой стремени \\
\hline Ost & Реконструкция между БП и головкой стремени \\
\hline Osd & БП напрямую репозиционирована к головке стремени \\
\hline Ofi & Реконструкция между наковальней и ножкой стремени \\
\hline Ofm & Реконструкция между молоточком и ножкой стремени \\
\hline Oft & Реконструкция между БП и ножкой стремени \\
\hline Ofd & БП напрямую репозиционирована к ножке стремени \\
\hline Ovi & $\begin{array}{c}\text { Реконструкция между наковальней и преддверием } \\
\text { (включая стапедотомию) }\end{array}$ \\
\hline Ovm & $\begin{array}{l}\text { Реконструкция между молоточком и преддверием } \\
\text { (маллеостапедотомия) }\end{array}$ \\
\hline Ovt & Реконструкция между БП и преддверием \\
\hline
\end{tabular}

выявили, что прогрессирование стадии заболевания ассоциировано с ухудшением слуха пациента.

В ряде исследований подтверждены прогностические значения стадии по EAONO/JOS в отношении улучшения слуха после операции $[8,21,22]$. В работе, проведенной A. Fukuda и соавт. (2019 г.) с включением 34 пациентов с ХСУ, показано, что восстановление слуха с достижением PTA-ABG $\leq 10$ и $\leq 20$ дБ после оперативного лечения наблюдается в 23,5 и $55,9 \%$ случаев соответственно, при этом более низкая стадия заболевания по системе стадирования EAONO/JOS статистически значимо ассоциирована с благоприятным исходом в отношении восстановления слуха [21]. В то же время в ретроспективном исследовании, проведенном H. van der Toom и соавт. (2020 г.), на достаточно большой выборке пациентов $(n=231)$ показано, что стадирование по EAONO/JOS уступает классификации STAMCO в отношении прогнозирования рецидирования заболевания [23]. Отсутствие прогностического значения в отношении рецидивирования стадии по EAONO/JOS также показано в исследовании, проведенном S. Angeli и соавт. (2020 г.) [24]. С этими данными согласуются и результаты исследования F. Ardic и соавт. (2020 г.): стадия по EAONO/JOS не позволяет прогнозировать риск рецидирования холестеатомы, но может использоваться как предиктор в отношении восстановления слуха после оперативного вмешательства и планирования оперативного вмешательства [8]. Также в работе, проведенной M. Eggink и соавт. (2021 г.), показано, что ни одна из предложенных систем стадирования холестеатомы (JOS, EAONO-JOS и STAMCO) не обладает достаточной точностью в отношении прогнозирования рецидирования заболевания, что требует дальнейших исследований [25].

\section{Классификация хирургических вмешательств на среднем ухе SAMEO-ATO}

В 2018 г. M. Yung и соавт. представили классификацию хирургических вмешательств на среднем ухе, разработанную под эгидой Международного общества по оценке результатов в отологии (The International Otology Outcome Group - IOOG) [26]. Разработка данной классификации проводилась на основании одного из методов принятия консенсусных решений в медицине - модифицированного метода Дельфи, который предполагает сбор мнений заинтересованных экспертов в данной области путем рассылки формализированных анкет с возможностью внесения комментариев. Первоначальный вариант классификации, разработанный группой из 8 экспертов, в последующем прошел несколько раундов обсуждения с представителями национальных отологических сообществ, а затем финальное обсуждение на специальной организованной сессии в рамках 31st Politzer Society Meeting (февраль 2018 г.). На этапе обсуждения с представителями отологических обществ уровень одобрения документа составил 95\%. Итоговая классификация, представляющая собой по сути систему формализации протоколов операций, получила название SAMEO-ATO ( $\mathrm{S}$ - state of surgery, A - approach, $\mathrm{M}$ - mastoidectomy, E - external ear canal reconstruction, $\mathrm{O}$ - obliteration of mastoid cavity, A - access to middle ear, $\mathrm{T}$ tympanic membrane, $\mathrm{O}$ - ossicular chain); табл. 1.

К настоящему времени представлены результаты нескольких клинических исследований, в которых использовалась классификация SAMEO-ATO для оценки риска осложнений и прогнозирования исходов оперативных вмешательств при ХСУ. В исследовании, проведенном V. Pontillo и соавт. (2021 г.), показано, что применение классификации SAMEO-ATO может быть полезно для прогнозирования улучшения слуха после операции. В это исследование включены 273 пациента, у большинства из которых (54\%) использовалась открытая хирургическая техника (Canal Wall Down, соответствует M2c по SAMEO-ATO), у 33\% - закрытая хирургическая техника (Canal Wall Up, соответствует 
M1b2a по SAMEO-ATO) [27]. Показано, что разделы M (mastoidectomy) и O (ossicular chain) системы формализации протоколов операции SAMEO-ATO ассоциированы с исходами в отношении слуха, при этом более неблагоприятные исходы выявлены для Мх и On [27]. Таким образом, в целом результаты этого исследования подтвердили значимость применения SAMEO-ATO для прогнозирования исходов операции. В другом исследовании, проведенном Т. Сассо и соавт. (2021 г.), показана значимость системы SAMEO-ATO для определения риска развития осложнений хирургического лечения. В данном исследовании проанализированы результаты 132 операций по поводу ХСУ у 110 пациентов, осложнения выявлены в $17 \%$ случаев (чаще всего - фистула латерального полукружного канала, реже - фистула верхнего полукружного канала и др.) [28]. По данным многомерного регрессионного анализа с риском развития осложнений статистически значимо ассоциированы оценка по SAMEOATO O1 (2 vs 1), SAMEO-ATO T (3 vs 1 или 2) и SAMEO-ATO O2 (S- vs F-) [28].

\section{Заключение}

ХСУ остается одной из наиболее актуальных проблем современной хирургической отологии. Для дальнейшего развития этого направления, унификации терминологии и описания протоколов хирургического лечения большое значение имеет внедрение единых классификаций, систем стадирования и формализации описания протоколов операций. В данной статье представлены новые классификации холестеатомы EAONO/JOS и хирургических вмешательств на среднем ухе SAMEO-ATO, проанализирован опыт их применения в рамках проведенных исследований. Показана возможность применения классификаций для определения прогноза в отношении восстановления слуха после операции. Широкое внедрение данных классификаций в практику может иметь большое значение для оптимизации сбора и анализа результатов хирургического лечения пациентов с ХСУ, определения предикторов эффективности разных методов лечения, риска развития осложнений и прогнозирования функционального исхода.

Раскрытие информации. Авторы декларируют отсутствие явных и потенциальных конфликтов интересов, связанных с публикацией настоящей статьи.

Disclosure. The authors declare that they have no competing interests.

Благодарность. Авторы выражают благодарность кафедре оториноларингологии ФГБОУ ДПО РМАНПО.

Gratitude. The authors would like to thank the otorhinolaryngology department of Russian Medical Academy of Continuous Professional Education.

\section{Литература/References}

1. Аникин М.И. Холестеатома среднего уха: определение, вопросы классификации и этиопатогенеза. Российская оториноларингология. 2016;82(3):16-23 [Anikin Ml. Kholesteatoma srednego ukha: opredelenie, voprosy klassifikatsii i etiopatogeneza. Rossiiskaia otorinolaringologiia. 2016;82(3):16-23 (in Russian)].
2. Rutkowska J, Özgirgin N, Olszewska E. Cholesteatoma Definition and Classification: A Literature Review. $J$ Int Adv Otol. 2017;13(2):266-71.

3. Aquino $\mathrm{JE}$, Cruz Filho NA, de Aquino JN. Epidemiology of middle ear and mastoid cholesteatomas: study of 1146 cases. Braz J Otorhinolaryngol. 2011;77:341-7.

4. Wallis S, Atkinson H, Coatesworth AP. Chronic otitis media. Postgrad Med. 2015;127(4):391-5.

5. Kuo CL. Etiopathogenesis of acquired cholesteatoma: prominent theories and recent advances in biomolecular research. Laryngoscope. 2015;125(1):234-40.

6. Yung M, Tono $\mathrm{T}$, Olszewska $\mathrm{E}$, et al. EAONO/JOS Joint Consensus Statements on the Definitions, Classification and Staging of Middle Ear Cholesteatoma. J Int Adv Otol. 2017;13(1):1-8.

7. Gaillardin L, Lescanne E, Morinière $S$, et al. Residual cholesteatoma: prevalence and location. Follow-up strategy in adults. Eur Ann Otorhinolaryngol Head Neck Dis. 2012;129:136-40.

8. Ardic FN, Mengi E, Tümkaya F, et al. Correlation between Surgical Outcome and Stage of Acquired Middle Ear Cholesteatoma: Revalidation of the EAONO/JOS Staging System. J Int Adv Otol. 2020;16(1):34-9.

9. Lien CF. Staging of attic cholesteatoma. Chin Med J. 1984;33:438-42.

10. Dornhoffer JL, Gardner E. Prognostic factors in ossiculoplasty: A statistical staging system. Otol Neurotol. 2001;22:299-304.

11. Black B, Gutteridge I. Aquired cholesteatoma: Classification and outcomes. Otol Neurotol. 2011;32:992-5.

12. Belal $A$, Reda $M$, Mehanna $A$, et al. TMC: A new staging system for tympanomastoid cholesteatoma. Egyptian J Otolaryngol. 2012;28:12-6.

13. Kitahara $T$, Mishiro $Y$, Sakagami $M$, et al. Staging-based surgical results in chronic otitis media with cholesteatoma. Nippon Jibiinkoka Gakkai Kaiho (Tokyo). 2012;115:91-100.

14. Olszewska E, Rutkowska J, Özgirgin N. Consensus-Based Recommendations on the Definition and Classification of Cholesteatoma. J Int Adv Otol. 2015;11:81-7.

15. Tono $T$, Sakagami $M$, Kojima $H$, et al. Staging and classification criteria for middle ear cholesteatoma proposed by the Japan Otological Society. Auris Nasus Larynx. 2017;44(2):135-40.

16. Komori $\mathrm{M}$, Tada $\mathrm{T}$, Koizumi $\mathrm{H}$, et al. Practical analysis of pars flaccida cholesteatoma with classification and staging system proposed by Japan Otological Society: a comparative study. Acta Otolaryngol. 2018;138(11):977-80.

17. Linder TE, Shah S, Martha AS, et al. Introducing the "ChOLE" Classification and Its Comparison to the EAONO/JOS Consensus Classification for Cholesteatoma Staging. Otol Neurotol. 2019;40(1):63-72.

18. James AL, Siu J, Borkhoff $\mathrm{CM}$, et al. International Collaborative Assessment of the Validity of the EAONO-JOS Cholesteatoma Staging System: Confirmation of Validity and Reliability. Otol Neurotol. 2020;41(2):286-7.

19. Sabour S. International Collaborative Assessment of the Validity of the EAONO-JOS Cholesteatoma Staging System: Methodological Issues on Validity and Reliability. Otol Neurotol. 2020;41(2):285.

20. Motegi M, Yamamoto $Y$, Tada $T$, et al. Clinical Characteristics of Pars Tensa Cholesteatoma: A Comparative Study of Area-Based Classification Systems Proposed by the Japanese Otological Society and the European Academy of Otology - Neuro-Otology. J Int Adv Otol. 2019;15(2):184-8.

21. Fukuda A, Morita S, Nakamaru $Y$, et al. Short-Term Hearing Prognosis of Ossiculoplasty in Pars Flaccida Cholesteatoma Using the EAONO/JOS Staging System. J Int Adv Otol. 2019;15(1):2-7.

22. Olmedo MJ, Ropero RF, Sánchez GS. Cholesteatoma: Influence of surgical technique and EAONO/JOS stage on audiological results. Acta Otorrinolaringol Esp. 2021:S0001-6519(21)00042-X.

23. Van der Toom $\mathrm{H}$, van der Schroeff MP, Janssen J, et al. A Retrospective Analysis and Comparison of the STAM and STAMCO Classification and EAONO/JOS Cholesteatoma Staging System in Predicting Surgical Treatment Outcomes of Middle Ear Cholesteatoma. Otol Neurotol. 2020;41(4):e468-74.

24. Angeli S, Shahal D, Brown CS, et al. Predicting Recidivism for Acquired Cholesteatoma: Evaluation of a Current Staging System. Otol Neurotol. 2020;41(10):1391-6.

25. Eggink MC, de Wolf MJF, Ebbens FA, et al. Evaluation of Long-term Cholesteatoma Recidivism: Using the JOS, EAONO-JOS, and STAMCO Cholesteatoma Staging Systems. Otol Neurotol. 2021;42(7):1031-8.

26. Yung $M$, James $A$, Merkus $P$, et al. International Otology Outcome Group and the International Consensus on the Categorization of Tympanomastoid Surgery. J Int Adv Otol. 2018;14(2):216-26.

27. Pontillo V, Damiani M, Graziano G, et al. Correlation between functional outcome and the SAMEO-ATO framework. Eur Arch Otorhinolaryngol. 2021. DOl:10.1007/s00405-021-07000-3

28. Cacco $T$, Africano $S$, Gaglio $G$, et al. Correlation between peri-operative complication in middle ear cholesteatoma surgery using STAMCO, ChOLE, and SAMEO-ATO classifications. Eur Arch Otorhinolaryngol. 2021. DOI:10.1007/500405-021-06679-8 